\title{
Maintenance Accessibility Evaluation Method based on D-H Model and Comfort in a Virtual Maintenance Environment
}

\section{Dong Zhou}

Beihang University

Hongduo Wu

Beihang University

Ziyue Guo ( $\nabla$ whd18811128802@163.com )

Beihang University

Qidi Zhou

Beihang University

Yuning Liang

Beihang University

\section{Research Article}

Keywords: Virtual maintenance, parameterization, accessibility evaluation, ergonomics, D-H model, RULA

Posted Date: September 7th, 2021

DOI: https://doi.org/10.21203/rs.3.rs-874075/v1

License: (9) This work is licensed under a Creative Commons Attribution 4.0 International License.

Read Full License 


\title{
Maintenance Accessibility Evaluation Method based on D-H Model and Comfort in a Virtual Maintenance Environment
}

\author{
Dong Zhou ${ }^{\mathrm{a}, \mathrm{b}, \mathrm{c}}$, Hongduo Wu ${ }^{\mathrm{a}, \mathrm{b}, \mathrm{c}}$, Ziyue Guo ${ }^{\mathrm{a}, \mathrm{b}, \mathrm{c}, *}$, Qidi Zhou ${ }^{\mathrm{a}, \mathrm{b}, \mathrm{c}}$, Yuning Liang ${ }^{\mathrm{a}, \mathrm{b}, \mathrm{c}}$ \\ ${ }^{a}$ School of Reliability and System Engineering, Beihang University, 100191, Beijing, China

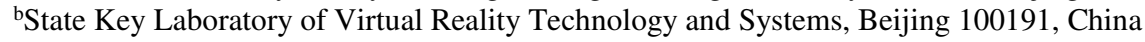 \\ 'State Key Defense Science and Technology Laboratory on Reliability and Environmental Engineering, Beijing 100191, China
}

\begin{abstract}
:
In virtual maintenance, the most widely used accessibility evaluation method is to use virtual human reach envelope surface to judge and evaluate accessibility. However, this method can only give two kinds of evaluation results: reachable and unreachable. There is not enough data and theoretical support for the construction of envelope surface, and the precision and accuracy of evaluation need to be improved. In this paper, a parameterized accessibility evaluation method and a method of accessibility envelope surface construction are proposed. Firstly, to objectively describe the movement of human body, a 6-joint and 5-link D-H (Denavit-Hartenberg) link model is established from the waist to the fingertip of the human body, and the ranges of 10 degrees of freedom and angles related to accessibility are determined according to ergonomics. Then, comfort is introduced to refine the accessibility evaluation, and a multi-level accessibility evaluation system based on comfort is constructed according to RULA (the rapid upper limb assessment). In order to facilitate the application of this method in virtual environment, a method of accessibility envelope surface construction is proposed. Firstly, the reachable points are generated by Monte Carlo simulation based on the D-H model. Secondly, the accessibility envelope surface is composed of the outermost random reachable points. Finally, the comparison experiment with the reachable envelope provided by DELMIA in virtual environment shows that the proposed method is more accurate, meticulous and objective. Based on this method, an accessibility evaluation tool has been developed in CATIA and has been applied in some scientific research institutes.
\end{abstract}

Keywords: Virtual maintenance, parameterization, accessibility evaluation, ergonomics, D-H model, RULA.

\section{Introduction}

Maintainability is the inherent property of the product. For large and complex products, maintainability is very important to the performance of products, operation cost and safety of use. Accessibility is the most important design index in the qualitative requirements of maintainability, and the first element of maintainability design analysis $[1,2]$. Accessibility refers to the degree of difficulty for maintenance personnel to reach maintenance objects. Good accessibility can make product maintenance convenient, fast, accurate and effective, and greatly save manpower, physical and time costs.

The early maintainability design works mainly rely on the physical prototypes or full-scale solid models of the product [3]. However, because these models are difficult to obtain in the product design stage, some necessary analysis and verification can not be carried out, which hinders the implementation of

Ziyue Guo's E-mail:whd18811128802@163.com design for manufacturing and assembly (DFMA) [4]. Virtual reality technology provides a means to carry out maintainability design analysis and verification based on virtual prototype. It can find the existing problems in the early stage of product design and give timely feedback for optimization, make up for the defects and deficiencies such as long cycle and difficult change, and provide a lot of data information for researchers to analyze. Therefore, the accessibility design and evaluation through virtual maintenance is a major trend of maintainability research. However, at present, the accessibility evaluation based on virtual maintenance technology is still based on qualitative evaluation, mainly through static verification and dynamic simulation. However, static verification has some defects, such as strong subjectivity and lack of theoretical support; dynamic simulation not only consumes a lot of time and energy to design the simulation process, but also the results of analysis and 
evaluation depend on the level of simulation designers, so the results are not accurate.

In the process of maintenance, the comfort of operation seriously affects the efficiency and effect of maintenance. Even if the maintenance target is reachable, the maintenance operation forces the maintenance personnel to maintain an uncomfortable maintenance posture, which is also a design defect of accessibility. Therefore, accessibility and comfort are two interrelated maintainability evaluation factors. The main idea of this paper is to refine and optimize the accessibility evaluation through comfort.

This manuscript is organized as follows. The second section discusses related work. The third part introduces the basic principle and steps of the parametric evaluation method of product entity accessibility based on comfort in virtual scene. In addition, the modeling process of accessibility evaluation model and the usage rules of evaluation tools are given. In the fourth part, a virtual maintenance case of airborne equipment is used to verify the effectiveness and superiority of the proposed method. Finally, the conclusions of this paper are given.

\section{Related Work}

\subsection{Virtual Maintenance}

Virtual maintenance technology can improve the level of maintainability design and shorten the design time and cost of equipment maintainability because of its advantages of simulating physical environment and obtaining space data effectively.

In the late 1980s, some foreign research institutions have begun to studied virtual simulation technology. In the design phase of F-16 fighter, in order to break through the limitation of physical prototype, LMTAS used virtual reality technology to find many maintainability problems and improve the scheme [5]. The University of Salford has established an immersive virtual platform considering constraints, which can effectively support virtual assembly [6,7]. Kallmann et al. studied the interaction characteristics of virtual interaction objects, and improved the timeliness and interactivity of virtual simulation ${ }^{[6]}$. Vujosevic et al. studied a new generation method of maintenance sequence. Through the combination of user interaction method and undirected closed graph method, the constraints are constantly updated to obtain a specific maintenance sequence [9]. Wampler et al. Proposed a new idea. They decomposed maintenance tasks and obtained a four layer decomposition model with the lowest layer being the macro motion layer. Simulation commands can be generated directly according to the macro motion layer and realize dynamic simulation [10].

China's research on virtual maintenance mainly focuses on static and dynamic simulation control for maintenance process and secondary development of DELMIA and Jack software to realize the required functions [11]. Wang et al. used DELMIA to carry out dynamic simulation of maintenance process, and analyze the maintainability of aircraft based on Simulation [12]. Wang Songshan et al. have studied the modeling and Simulation of virtual digital prototype and human interaction behaviour, established the relationship with product CAD system, and realized the development of related functions in JACK [13]. Li Xinxing et al. decompose the maintenance task to the maintenance dynamic element level, and get the maintenance task decomposition model. The use of maintenance kinesiology can better describe people's sports behaviour [14]. LV Chuan and other scholars from Beijing University of Aeronautics and Astronautics used the hybrid control method to control the action of virtual human, combined the peripheral control method with the algorithm control method, and made full use of their advantages to provide a new means for realizing the accurate control of virtual human action [15]. Zhou Dong and other scholars have established a new maintenance activity simulation management model MTN (Maintenance Task Net), which combines the abstract description of maintenance activities with the underlying activities of dynamic simulation, and can drive the virtual environment and virtual human to simulate maintenance tasks [16]. In order to get rid of the constraints that virtual maintenance simulation on digital mockups (DMUs) requires a lot of time and professional maintenance knowledge, Guo ziyue and others proposed an immersive maintenance simulation method in virtual environment based on virtual reality, and comprehensively evaluated the product maintainability state by using fuzzy comprehensive evaluation (FCE) [17]. Yang Yingyan et al. divided the virtual maintenance dynamic simulation process into two parts: simulation preparation stage and simulation generation stage, and defined the main work of each stage to better carry out maintenance simulation [18].

To sum up, the research on virtual maintenance technology mainly focuses on the development of virtual maintenance platform, virtual digital prototype modeling, virtual human modeling, simulation command generation and human motion synthesis [18]. However, there are three main problems: 1) for the modeling of complex virtual digital prototype, especially flexible cable, the current technology is still very limited and needs further research; 2) In dynamic simulation, the authenticity of virtual human action has been questioned, and the maintainability analysis and 
evaluation results are not very accurate. Therefore, it is necessary to study the virtual human action control method to improve the action credibility; 3) Designing virtual dynamic simulation has always been a cumbersome and complex thing, which greatly affects the work efficiency of designers. How to realize the rapid synthesis of virtual human actions, how to realize the automatic generation of simulation process, and even how to improve the static virtual maintainability evaluation effect without generating maintenance actions are aspects that need technical breakthrough.

\subsection{Accessibility Analysis and Evaluation}

Around 1950, the concept of maintenance accessibility was proposed by some American scholars. Maintenance accessibility refers to the difficulty of approaching the parts to be repaired when repairing the product [19]. At present, many related engineering software have been developed abroad. DEPTH and CREW CHIEF developed by the US air force can analyze the maintainability of products by using virtual mannequins to help researchers analyze the relationship and interaction between maintenance personnel and systems [20]. American RC has developed a software BlockSim that can analyze and calculate reliability and maintainability, which can describe the system through reliability block diagram [21]. The IVAS system designed by the State Key Laboratory of Zhejiang University can establish a very real virtual environment and has powerful interactive means to support virtual people to carry out virtual process simulation in it, which plays a good auxiliary role in the whole life cycle of product equipment development and the realization of concurrent engineering [22]. The Fifth Institute of electronics of the Ministry of information industry of China has designed a software named CARMES for reliability design, analysis and management, which has well supported the reliability growth and safety guarantee of Shenzhou V spacecraft [23]. In 2003, the design of China's first aircraft electronic digital prototype was completed. The prototype has established a complete product digital standard system, which can check and analyze the maintenance accessibility, interference and personnel analysis in the maintainability analysis [24]. Guo et al. Proposed an immersive maintainability verification and evaluation system (IMVES) based on virtual reality, which enables users to interact with maintenance objects and conduct immersive simulation, so as to solve the problem that accessibility evaluation through non-immersive virtual maintenance simulation is very cumbersome and time-consuming [25].

Up to now, there are two kinds of methods to evaluate the accessibility based on virtual maintenance. One is to evaluate the accessibility by designing and analyzing the simulation process. The process of maintenance simulation not only costs a lot of energy, but also is not necessarily true. In addition, the evaluation process depends on expert experience, which is subjective. Another accessibility evaluation method is to use the virtual human accessibility envelope to judge whether the target is reachable. For example, the accessibility analysis tool provided by DELMIA software developed by Dassault company of France is shown in Fig. 1. Engineers can get the evaluation result of maintenance accessibility by observing the relative position relationship between maintenance target and arm envelope ball. However, this method can only give two evaluation results: reachable and unreachable. In addition, the construction of reachable envelope surface lacks sufficient data and theoretical support, and the accuracy and accuracy of evaluation need to be improved.

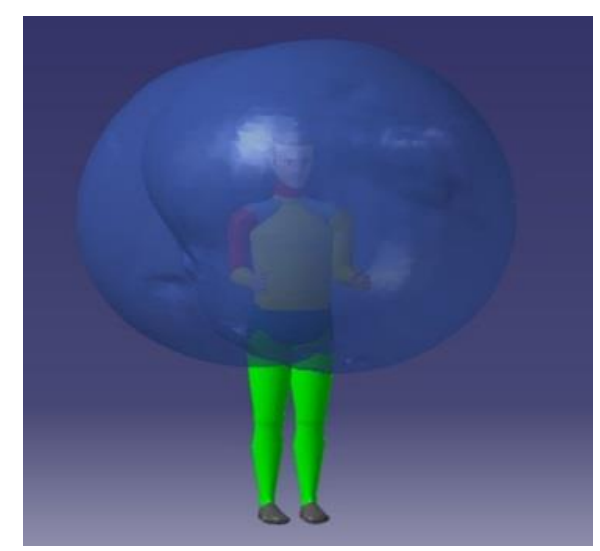

Fig. 1. Virtual human hands reachable space envelope ball in DELMIA

\subsection{Evaluation method of human comfort}

At present, the methods of human comfort evaluation are mainly obtained through the experimental statistics. There are three widely used methods: OWAS (Ovako Working Posture Analysis) [26], RULA (Rapid Upper Limb Assessment) [27], LUBA (Loading on the upper body Assessment) [28]。

OWAS mainly analyzes and evaluates people's body posture at work, and carries out grading evaluation according to the possible degree of injury caused by working posture. OWAS method mainly encodes the weight of four parts of human body: head and neck, trunk, arm, leg and human load. Through a large number of experimental observation and verification, the researchers obtained the comfort parameters of each body posture, and divided it into four levels according to the harm of posture to the body, so as to evaluate the staff's posture. The different place between RULA and OWAS is to grade the posture of the limbs by scoring. Compared with the coding classification, the method can score according to the angle of different joints, so as to get the final posture 
score. LUBA establishes a relationship between each upper limb posture and its maximum working duration, which is more suitable for the scene of maintaining a posture for a long time.

\section{Proposed Method}

In this paper, accessibility and comfort are regarded as two interrelated maintainability evaluation factors. Therefore, this paper introduces comfort, refines and optimizes the accessibility evaluation based on objective data and mathematical model, proposes a rapid maintainability grading evaluation method in virtual environment, and develops an accessibility evaluation tool based on this method, which can be applied in DELMIA and CATIA. The implementation process of the accessibility parametric evaluation method proposed in this paper is shown in Fig. 2.

The idea of this paper is to build the human linkage model and inject the kinematic model constrained by comfort to realize the parametric grading evaluation of accessibility. Firstly, we need to analyze and collect the information and data needed for accessibility evaluation in virtual environment; secondly, we use simplified human body model and D$\mathrm{H}$ parameter method to build human kinematics model; secondly, we classify the accessibility and determine the evaluation system from the perspective of comfort based on RULA; for the convenience of use, we develop a multi-level accessibility evaluation model tool based on the proposed method; Finally, the effectiveness and superiority of the method are verified in an airborne virtual environment.

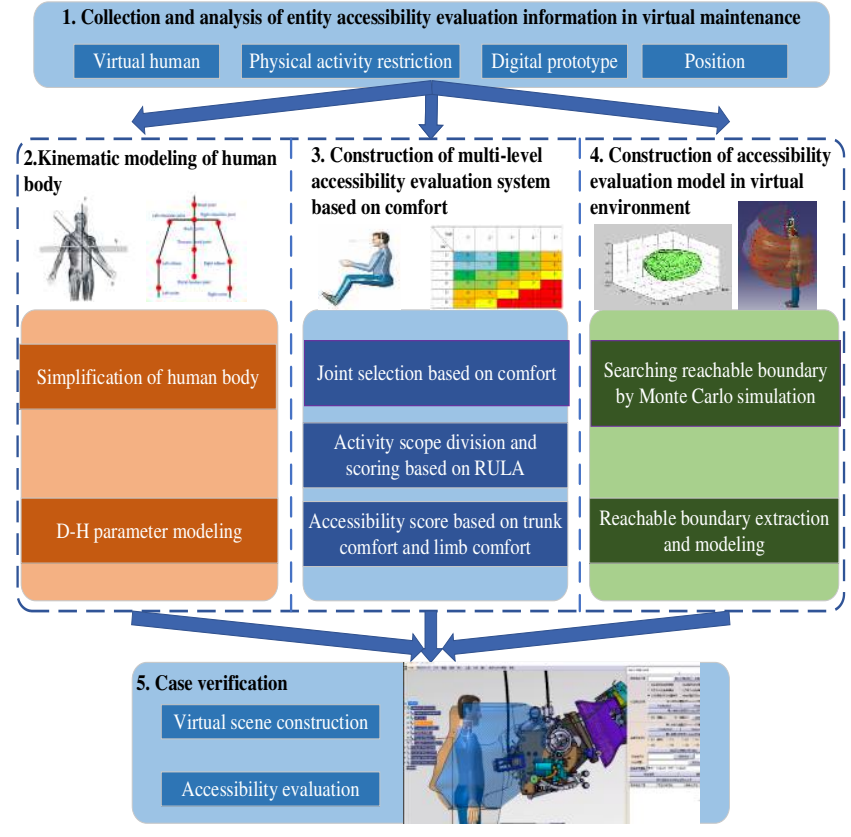

Fig. 2 The method structure diagram of this paper

\subsection{Data Analysis of Virtual Maintenance Eccessibility Evaluation}

Compared with the real environment, the important advantage of virtual environment is that there are a lot of data in virtual environment for researchers to use, so it is necessary to determine the data needed for accessibility analysis in virtual environment.

In the virtual environment of maintenance operation, the reachability analysis needs to be judged according to the reachable range of limbs and the spatial position of the maintenance object. Therefore, it is necessary to understand the virtual human modeling technology and obtain the motion constraints of each limb structure of the virtual human body, including the length of the limb and the range of motion of the included joint degrees of freedom, so as to construct the reachable envelope model of the limb and obtain the reachability of the limb range. In the evaluation stage, accessibility is the maintainability evaluation factor generated by the relative distance between human and maintenance object. Therefore, it is necessary to obtain not only the spatial location information of virtual human and maintenance object, but also the virtual human model and digital prototype of maintenance object, that is, the data of size and shape, so as to analyze and evaluate the accessibility.

\subsection{Kinematic Modelling of Human Body}

The human body is a very complex structure, in order to facilitate the study of human motion, we need to build a human model. Different human models have different ways of movement, among which H-Anim human model is widely accepted and applied in various 
fields [29]. According to the characteristics of human body, H-Anim model obtains the data of each joint node of human body model, and establishes the node database, including various types of information such as attitude parameters, degree of freedom parameters and so on. There are 77 joints and 47 bone segments in $\mathrm{H}$-Anim model, and each bone is connected by joint [30]. However, for the common maintenance activities, the H-Anim model contains too many joints and bone segments, and there is a lot of redundancy, so it needs to be simplified to get the manikin suitable for maintenance activities in virtual environment.

\subsubsection{Simplification of Human Body}

The accessibility discussed in this paper is on the premise that the maintenance personnel have been standing in the best maintenance position, so the impact of the accessibility caused by the joints below the waist is not discussed.

The joints and limbs above the waist were simplified and the linkage model was reconstructed. Firstly, a standard link model is established from waist to fingertip, and the nodes are waist-chest-neckshoulder-elbow-wrist-fingertip. Among them, waist can rotate and tilt, chest and neck movement can not affect the range of hand movement, but from shoulder to fingertip a series of nodes can affect accessibility. In addition, because this paper only considers the maximum envelope range that the arm can reach, and does not consider the specific motion of the hand, the hand can be regarded as a bone structure, that is, a rigid link. The simplified human model of the upper part of the body is shown in Fig. 3, and the red nodes are reserved nodes.

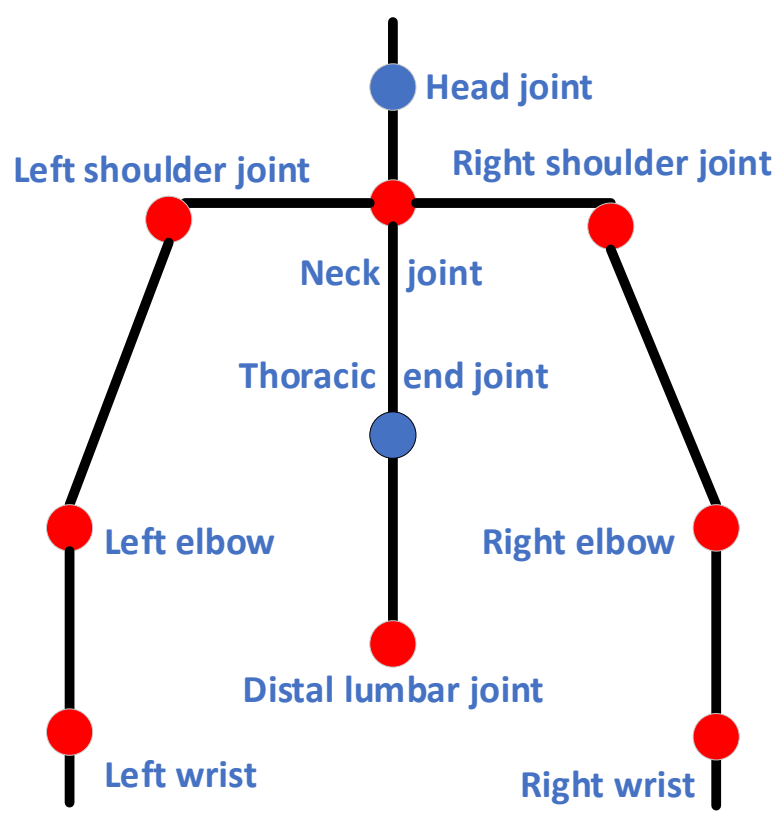

Fig. 3 Simplified manikin(the upper part of the body)

\subsubsection{D-H Parameter Modelling}

In order to study the relationship between the joints and degrees of freedom of the arm in motion, this paper uses the Denavit-Hartenberg parameter method for limb kinematics modelling [31]. The D-H parameter method can express the motion relationship between the connecting rods, so as to deduce the motion relationship of the end of the limb relative to the starting end. The construction of human D-H model can be divided into two steps.

\section{(1) Analysis of Degree of Freedom}

The degree of freedom of joints determines the way and direction of limb movement. The degrees of freedom of five joints from waist to wrist are discussed as follows.

- Waist (three degrees of freedom): trunk forward and backward, trunk left and right, rotation;

- Neck (no degree of freedom): no degree of freedom affecting accessibility;

- Shoulder (three degrees of freedom): arm up and down, arm forward and backward, rotation;

- Elbow (two degrees of freedom): bend, rotate;

- Wrist (two degrees of freedom): Bend to both sides, Bend back and forth.

The model has ten degrees of freedom in five joints, and the schematic diagram is shown in Fig. 4, where $Z_{i}(i=0,2, \ldots, 9)$ represents the degrees of freedom, $L_{j}(j=1,2, \ldots, 5)$ represents the length of trunk and limb.

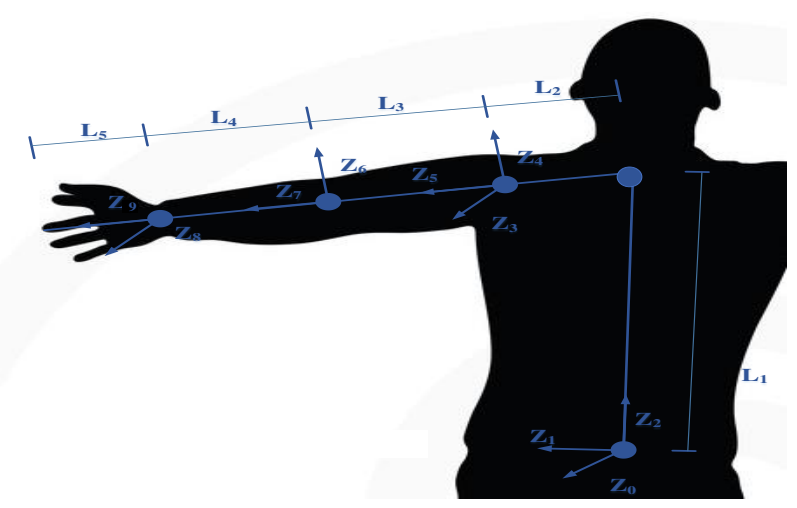

Fig. 4. Degree of freedom division of each joint of human body model

\section{(2) Establishment of Limb Kinematics Model based on D-H Parameter Method}

The D-H parameter method establishes a coordinate system for each degree of freedom, describes the relative motion relationship between joints by the 
change matrix between coordinate systems, and controls the angle and range of motion. The change matrix of coordinate system $i$ relative to coordinate system $i-1$ is shown in Eq. (1).

$$
{ }_{i}^{i-1} T=\left[\begin{array}{cccc}
c \theta_{i} & -c \alpha_{i} s \theta_{i} & s \alpha_{i} s \theta_{i} & a_{i} c \theta_{i} \\
s \theta_{i} & c \alpha_{i} c \theta_{i} & -s \alpha_{i} c \theta_{i} & a_{i} s \theta_{i} \\
0 & s \alpha_{i} & c \alpha_{i} & d_{i} \\
0 & 0 & 0 & 1
\end{array}\right]
$$

Where, $s \theta_{i}$ represents $\sin \theta_{i}$; s $\alpha i$ represents $\sin \alpha_{i}$; $c \theta_{i}$ represents $\cos \theta_{i}$; $c \alpha i$ represents $\cos \alpha_{i} ; a_{i}$ is the length of the rod, which represents the distance between the two connected joint axes; $\alpha_{i}$ is the angle, which means the angle of $x_{i}$ axis on degree of freedom $i$ rotating from $z_{i-1}$ axis to $z_{i}$ axis according to the righthand law; $d_{i}$ is the translation, representing the distance difference between adjacent connecting rods on the joint axis, and the positive direction of $z_{i-1}$ axis is its positive direction; $\theta_{i}$ is the amount of rotation, which indicates the angle of $z$ axis rotation from $x_{i-1}$ axis to $x_{i}$ axis according to the right-hand rule.

Then, the parameters of D-H linkage model are determined according to ergonomics, Chinese National Institute of Standards GB10000-1988 [32] (human parameters of different countries and regions can be referred to other data) and experiments, including the motion angle of each degree of freedom, the length between each joint (rod length), as shown in Table 1 and 2.

Table 1 D-H model parameter table

\begin{tabular}{c|c|c|c|c|c}
\hline $\boldsymbol{i}$ & $\boldsymbol{Z}_{\boldsymbol{i}-\boldsymbol{1}}$ & $\boldsymbol{a}_{\boldsymbol{i}}$ & $\boldsymbol{\alpha}_{\boldsymbol{i}}$ & $\boldsymbol{d}_{\boldsymbol{i}}$ & $\boldsymbol{\theta}_{\boldsymbol{i}}$ \\
\hline 1 & $\mathrm{Z}_{0}$ & 0 & $\pi / 2$ & 0 & $\theta_{1}$ \\
\hline 2 & $\mathrm{Z}_{1}$ & 0 & $\pi / 2$ & 0 & $\theta_{2}$ \\
\hline 3 & $\mathrm{Z}_{2}$ & $\mathrm{~L}_{2}$ & $-\pi / 2$ & $\mathrm{~L}_{1}$ & $\theta_{3}$ \\
\hline 4 & $\mathrm{Z}_{3}$ & 0 & $\pi / 2$ & 0 & $\theta_{4}$ \\
\hline 5 & $\mathrm{Z}_{4}$ & 0 & $\pi / 2$ & 0 & $\theta_{5}$ \\
\hline 6 & $\mathrm{Z}_{5}$ & 0 & $\pi / 2$ & $\mathrm{~L}_{3}$ & $\theta_{6}$ \\
\hline 7 & $\mathrm{Z}_{6}$ & 0 & $\pi / 2$ & 0 & $\theta_{7}$ \\
\hline 8 & $\mathrm{Z}_{7}$ & 0 & $\pi / 2$ & $\mathrm{~L}_{4}$ & $\theta_{8}$ \\
\hline 9 & $\mathrm{Z}_{8}$ & 0 & $\pi / 2$ & 0 & $\theta_{9}$ \\
\hline 10 & $\mathrm{Z}_{9}$ & $\mathrm{~L}_{5}$ & 0 & 0 & $\theta_{10}$ \\
\hline
\end{tabular}

Table 2 Movement angle of each degree of freedom

\begin{tabular}{c|c|c}
\hline Number & Scale & Illustration \\
\hline$\theta_{l}$ & $-10^{\circ} \sim 37^{\circ}$ & $\begin{array}{c}\text { The inclination of the } \\
\text { upper body forward or } \\
\text { backward from the }\end{array}$ \\
\hline
\end{tabular}

\begin{tabular}{|c|c|c|}
\hline & & waist \\
\hline$\theta_{2}$ & $-8^{\circ} \sim 8^{\circ}$ & $\begin{array}{l}\text { The inclination of the } \\
\text { upper body from the } \\
\text { waist to the left or right }\end{array}$ \\
\hline$\theta_{3}$ & $-10^{\circ} \sim 10^{\circ}$ & $\begin{array}{c}\text { Rotation angle of } \\
\text { human upper body from } \\
\text { waist }\end{array}$ \\
\hline$\theta_{4}$ & $-45^{\circ} \sim 180^{\circ}$ & $\begin{array}{l}\text { The angle at which the } \\
\text { upper arm is raised or } \\
\text { lowered }\end{array}$ \\
\hline$\theta_{5}$ & $-40^{\circ} \sim 90^{\circ}$ & $\begin{array}{c}\text { The angle at which the } \\
\text { upper arm is moved } \\
\text { outward or inward }\end{array}$ \\
\hline$\theta_{6}$ & $-45^{\circ} \sim 90^{\circ}$ & $\begin{array}{l}\text { The rotation angle of } \\
\text { shoulder joint with the } \\
\text { upper arm as the axis }\end{array}$ \\
\hline$\theta_{7}$ & $0^{\circ} \sim 140^{\circ}$ & $\begin{array}{c}\text { The angle between } \\
\text { upper and lower arms at } \\
\text { elbow }\end{array}$ \\
\hline$\theta_{8}$ & $-70^{\circ} \sim 80^{\circ}$ & $\begin{array}{l}\text { The rotation angle of } \\
\text { the elbow joint with the } \\
\text { forearm as the axis }\end{array}$ \\
\hline$\theta_{9}$ & $-30^{\circ} \sim 50^{\circ}$ & $\begin{array}{l}\text { At the wrist joint, the } \\
\text { angle at which the palm } \\
\text { bends to the side } \\
\text { (thumb side or little } \\
\text { thumb side) }\end{array}$ \\
\hline$\theta_{10}$ & $-90^{\circ} \sim 80^{\circ}$ & $\begin{array}{l}\text { At the wrist joint, the } \\
\text { angle at which the palm } \\
\text { bend to the front and } \\
\text { back (palm side or back } \\
\text { side of the hand) }\end{array}$ \\
\hline
\end{tabular}

The relative position change relationship (one-step change matrix) between adjacent degrees of freedom is determined by the above parameters, and the relative position change relationship of leaf node (finger) relative to root node (waist) is obtained by multiplying multiple one-step change matrices, as shown in Eq. (2).

$$
{ }_{10}^{0} T\left(\theta_{1}, \theta_{2}, \ldots, \theta_{n}\right)={ }_{1}^{0} T\left(\theta_{1}\right){ }_{2}^{1} T\left(\theta_{2}\right) \ldots{ }_{10}^{9} T\left(\theta_{n}\right)
$$

Let $P$ denote the position vector of the beginning of the model in the coordinate system $Z_{0}$, and $X$ denote the position vector of the end of the model. Then the relation between $X$ and $P$ is expressed as Eq. (3). 


$$
\left[\begin{array}{c}
X \\
1
\end{array}\right]={ }_{\mathrm{n}}^{0} T\left[\begin{array}{c}
P \\
1
\end{array}\right]={ }_{1}^{0} T{ }_{2}^{1} T \ldots{ }_{\mathrm{n}}^{n-1} T\left[\begin{array}{c}
P \\
1
\end{array}\right]
$$

The equation describes the motion relationship of the finger relative to the waist, which is the basis of establishing the reachable envelope surface model.

\subsection{Construction of Multi-level Accessibility Evaluation System Based on Comfort}

Comfort and accessibility are two inseparable characteristics in maintenance operation. This paper establishes a relationship between comfort and accessibility based on RULA.

\subsubsection{Joint Freedom Degrees Selection based on Comfort}

In order to improve the practicability of the accessibility evaluation method and reduce the workload of modelling, it is necessary to select the degree of freedom that affects the comfort from the 10 included in the above D-H model

In this paper, the degrees of freedom involved in some limb comfort evaluation methods are selected. At present, there are three widely used methods: OWAS, RULA and LUBA. Most of the joints involved in the three methods are concentrated in the waist, shoulder and elbow, and considering that the rotation of shoulder and elbow has little effect on the reachable range, a total of 6 degrees of freedom $Z_{0}, Z_{1}, Z_{2}, Z_{3}, Z_{4}$, $\mathrm{Z}_{7}$ in Fig. 4 are selected.

\subsubsection{Activity Scope Division and Score based on RULA}

Although OWAS, RULA and LUBA the three comfort evaluation methods all adopt the idea of dividing posture, OWAS method divides posture roughly and does not divide the angle of limbs; LUBA method considers the duration of working posture more, and the classification of posture is only three levels. RULA divides the range of motion of the arm and trunk, and gives the corresponding score. Therefore, this paper divides the range of the selected six degrees of freedom based on RULA (Before dividing the angle range, intersect the angle range specified in RULA with the range specified in Table 2) and defines the corresponding comfort score. In this paper, the comfort is divided into "arm comfort" and "trunk comfort". The arm comfort score is the sum of the three degrees of freedom comfort scores of shoulder and elbow, and the trunk comfort score is the sum of the three degrees of freedom comfort scores of waist. The motion range and corresponding score of each degree of freedom are shown in Table 3.

\begin{tabular}{|c|c|c|c|}
\hline \multicolumn{4}{|c|}{ Arm comfort score } \\
\hline Limbs & $\begin{array}{c}\text { Description of } \\
\text { movement }\end{array}$ & Angle & Score \\
\hline \multirow{8}{*}{ Upper Arm } & \multirow{3}{*}{$\begin{array}{c}\text { Stretch out or } \\
\text { close inward } \\
\left(\theta_{5}\right)\end{array}$} & $\theta_{5}=0^{\mathbf{o}}$ & 0 \\
\hline & & $0^{\circ} \leq \theta_{5}<90^{\circ}$ & \multirow{2}{*}{1} \\
\hline & & $-45^{\circ}<\theta_{5} \leq 0^{\circ}$ & \\
\hline & \multirow{5}{*}{$\begin{array}{l}\text { Raise or lower } \\
\qquad\left(\theta_{4}\right)\end{array}$} & $\begin{array}{c}-20^{\circ} \leq \theta_{4} \leq \\
20^{\circ}\end{array}$ & 1 \\
\hline & & $\begin{array}{c}-40^{\circ} \leq \theta_{4}<- \\
20^{\circ}\end{array}$ & 2 \\
\hline & & $20^{\circ}<\theta_{4} \leq 45^{\circ}$ & \\
\hline & & $45^{\circ}<\theta_{4} \leq 90^{\circ}$ & 3 \\
\hline & & $\begin{array}{c}90^{\circ}<\theta_{4} \leq \\
120^{\circ}\end{array}$ & 4 \\
\hline \multirow{3}{*}{ Lower arm } & \multirow{3}{*}{$\begin{array}{l}\text { The angle } \\
\text { between the } \\
\text { upper arm and } \\
\text { the lower } \\
\left(\theta_{7}\right)\end{array}$} & $60^{\circ} \leq \theta_{7} \leq 100^{\circ}$ & 0 \\
\hline & & $0^{\circ} \leq \theta_{7}<60^{\circ}$ & \multirow[b]{2}{*}{1} \\
\hline & & $\begin{array}{c}100^{\circ}<\theta_{7} \\
\leq 130^{\circ}\end{array}$ & \\
\hline \multicolumn{4}{|c|}{ Trunk comfort score } \\
\hline Trunk & $\begin{array}{c}\text { Description of } \\
\text { movement }\end{array}$ & Angle & Score \\
\hline \multirow{10}{*}{ Waist } & \multirow{4}{*}{$\begin{array}{c}\text { Lean forward } \\
\text { or backward } \\
\qquad\left(\theta_{l}\right)\end{array}$} & $\theta_{l}=0^{\circ}$ & 1 \\
\hline & & $0^{\circ} \leq \theta_{1}<20^{\circ}$ & \multirow{2}{*}{2} \\
\hline & & $-10^{\circ}<\theta_{1} \leq 0^{\circ}$ & \\
\hline & & $20^{\circ} \leq \theta_{1}<37^{\circ}$ & 3 \\
\hline & \multirow{3}{*}{$\begin{array}{l}\text { Lean left or } \\
\text { right } \\
\left(\theta_{2}\right)\end{array}$} & $\theta_{2}=0^{\circ}$ & 0 \\
\hline & & $-8^{\circ} \leq \theta_{2}<0^{\circ}$ & \multirow{2}{*}{1} \\
\hline & & $0^{\circ}<\theta_{2} \leq 8^{\circ}$ & \\
\hline & \multirow{3}{*}{$\begin{array}{l}\text { Rotation } \\
\qquad\left(\theta_{3}\right)\end{array}$} & $\theta_{3}=0^{\circ}$ & 0 \\
\hline & & $-10^{\circ} \leq \theta_{3}<0^{\circ}$ & \multirow{2}{*}{1} \\
\hline & & $0^{\circ}<\theta_{3} \leq 10^{\circ}$ & \\
\hline
\end{tabular}

\subsubsection{Accessibility Score based on Trunk Comfort and Limb Comfort}

The multi-level accessibility evaluation system is constructed by the arm comfort score and trunk comfort score, and is represented by the matrix two parameter comfort evaluation table. The accessibility score of each case is determined by fuzzy comprehensive evaluation method, among which, the evaluation factor theory field is "arm comfort" and "trunk comfort", with the same weight, each of which is 0.5 ; the evaluation grade theory field is composed of 2 to 8 points, including 7 grades; the fuzzy evaluation vector synthesis operator is matrix multiplication; the determination of the score adopts the principle of maximum membership. The comfort evaluation table is shown in Table 4, with a score of 1-8, 1 point for not reachable, and 8 points for the maintenance target location in the most comfortable accessible area.

Table 4 Comprehensive evaluation table of accessibility based on comfort

Table 3 Comfort score table 


\begin{tabular}{c|c|c|c|c|c}
\hline Trunk & 1 & 2 & 3 & 4 & 5 \\
\hline 1 & 8 & 7 & 6 & 5 & 4 \\
\hline 2 & 7 & 7 & 6 & 5 & 4 \\
\hline 3 & 6 & 5 & 5 & 4 & 3 \\
\hline 4 & 5 & 5 & 4 & 3 & 2 \\
\hline 5 & 4 & 4 & 3 & 2 & 2 \\
\hline 6 & 4 & 3 & 2 & 2 & 2 \\
\hline
\end{tabular}

Up to now, this method can support accessibility evaluation in virtual maintenance. However, considering that every evaluation needs to readjust the virtual human's posture and obtain the required data, and it is found that the reachability range corresponding to each reachability score in Table 5 is fixed, so it is more convenient to establish the reachability envelope model of the reachability range corresponding to each reachability score in simulation software such as DELMIA or CATIA.

\subsection{Construction of Accessibility Evaluation Model in Virtual Environment}

The accessibility envelope model of virtual human is the main means of accessibility evaluation in virtual maintenance. In view of the multi-level accessibility evaluation system established in this paper, the D-H model established above can be used to search the reachable range corresponding to each score by simulation, so as to establish the reachable envelope surface model. This paper also gives the use criteria of the accessibility evaluation model in virtual environment.

\subsubsection{Searching Reachable Boundary}

Eq. (3) describes the motion constraint relationship of fingers relative to the waist. After setting the limb length and the angle of movement, the position of the fingers relative to the waist can be calculated. Therefore, for the D-H model established above, input the length of limbs and trunk and other parameters (the data comes from the Chinese national standard GB10000-1988), adjust the range of motion of six degrees of freedom related to comfort in the model according to the accessibility score of each level in Table 4, and simulate the activity process from waist to fingertip through Monte Carlo simulation, so as to generate the accessibility range In the process of Monte Carlo simulation, the other four degrees of freedom which have little relationship with comfort are set as the maximum range (Table 2). The search process for the reachable boundary from waist to fingertip is as follows.
Input: The value or range of parameters needed in $\mathrm{D}-\mathrm{H}$ model;

Step1: Determine the location of root node(anywhere);

Step2: For each accessibility score in Table 5, find out each possible range of motion of six degrees of freedom related to comfort (for example, for 8 points of accessibility, corresponding to 1 point of arm comfort and 1 point of trunk comfort, where the parameter range set corresponding to 1 point of arm comfort is $\left\{\theta_{1}=0^{\circ},-20^{\circ} \leq \theta_{2} \leq 20^{\circ}, 60^{\circ} \leq \theta_{3} \leq 100^{\circ}\right\}$, the range set of parameters corresponding to 1 score of trunk comfort is $\left.\left\{\theta_{4}=0^{\circ}, \theta_{5}=0^{\circ}, \theta_{7}=0^{\circ}\right\}\right)$

Step3: For each parameter with a certain range of values, enough random numbers are generated in the range of values;

Step4: According to Eq. (3), several reachable points are generated by Monte Carlo simulation;

Step5: Repeat step 2 to step 4 to search the reachable point set with 2 to 8 points;

Output: The coordinates of each point in the accessible point set of 2 to 8 points.

\subsubsection{Reachable Boundary Extraction and Modeling}

Firstly, we need to extract the boundary points of the reachable point set by extracting convex package to get the reachable range. The process of boundary extraction is shown in Fig. 5.

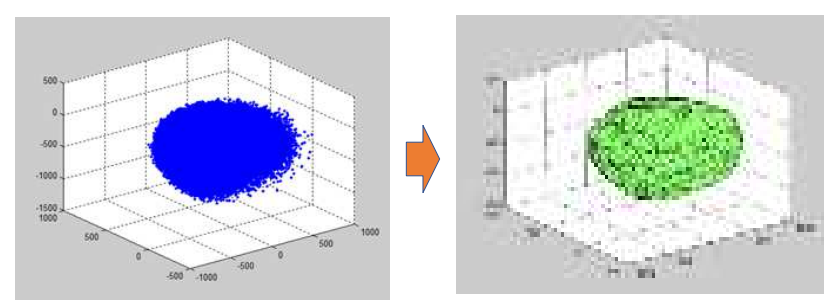

Fig. 5 Extracting the boundary of reachable point set

Based on the extracted reachable boundary, the reachable envelope model can be built in CATIA, UG, 3DMax etc. In order to ensure the authenticity and beauty of the model, the reachable envelope model needs to be smoothed. So far, the reachable envelope model from waist to one hand has been established, and the model of the other hand can be generated according to the axial symmetry. The reachable envelope model of two hands with 2 to 8 points of accessibility is shown in Fig. 6. 


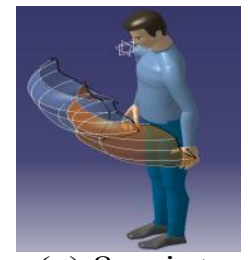

(a) 8 points

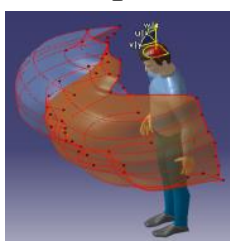

(d) 5 points

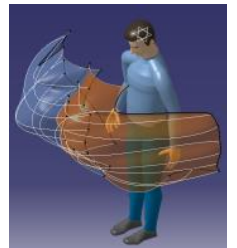

(b) 7 points

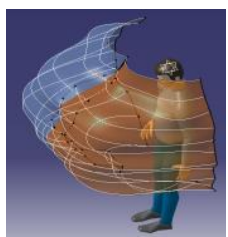

(e) 4 points

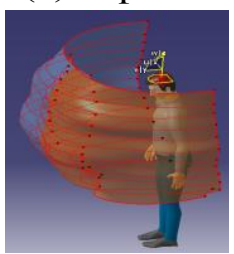

(g) 2 points

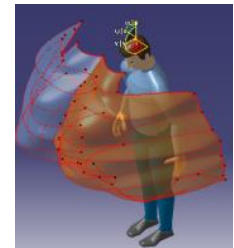

(c) 6 points

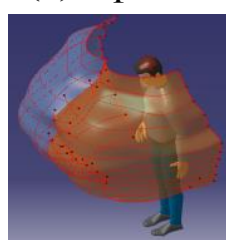

(f) 3 points

Fig. 6 Reachable envelope model with accessibility score of 2 to 8

Based on the above model, a parameterized accessibility evaluation tool which can run under DELMIA is developed. The method of using the tool is as follows: import virtual human and the human accessibility envelope models from large to small into the virtual maintenance scene through the interactive interface; adjust the virtual human to the best maintenance position according to the location of the maintenance target; if the accessibility envelope of a certain score $K$ completely covers the target point and the accessibility envelope of the score $K+1$ cannot completely cover the target point, then the accessibility score of the verification point is $K$. The accessibility evaluation results of this evaluation can be recorded and exported in the tool.

\section{Case Verification}

This paper applies the accessibility evaluation tool developed based on the proposed method to the accessibility evaluation of APU of a wide body passenger aircraft. This case mainly aims at the accessibility evaluation of the maintenance interface involved in the disassembly of the starter APU.

\subsection{Construction of Virtual Maintenance Scene}

Building virtual maintenance scene is the basis of virtual maintenance evaluation. Create a process in DELMIA and import the ground, virtual human, maintenance objects, maintenance tools, etc., and according to the maintenance process, place the maintenance personnel, maintenance objects and other facilities in the maintenance scene as truly as possible, as shown in Fig 7.

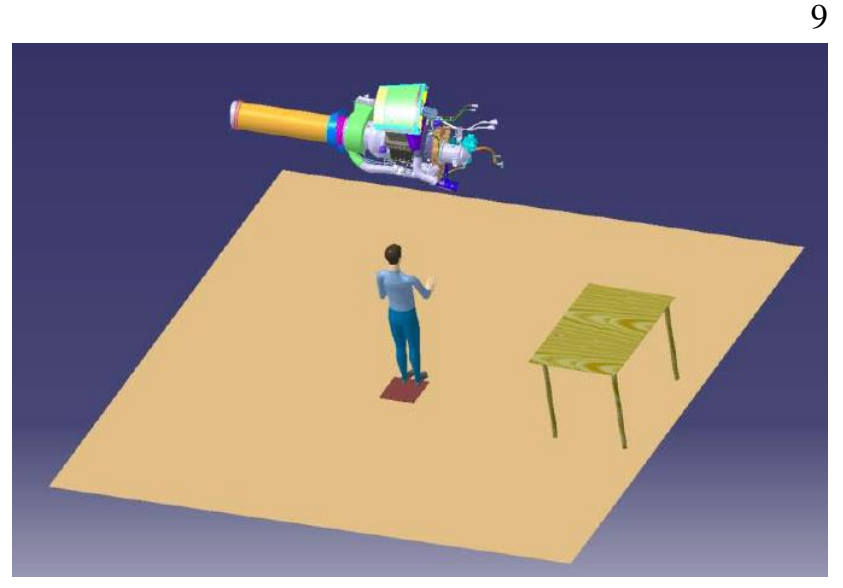

Fig. 7 Build a virtual maintenance environment

In this case, accessibility evaluation is carried out for the disassembly process of APU starter. The specific maintenance process is to dismantle 8 fastening bolts around the starter. Therefore, these bolts are the main objects of accessibility evaluation in this case, as shown in Fig. 8.

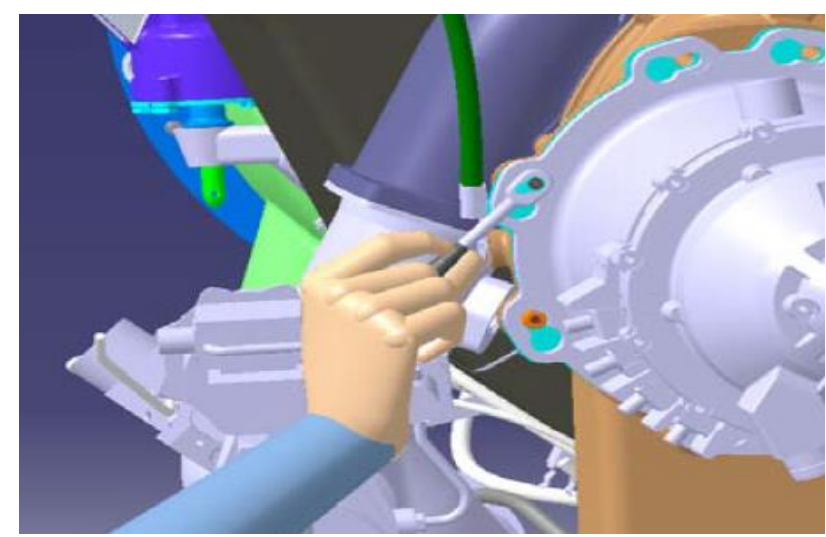

Fig. 8 Maintenance targets and location

\subsection{Accessibility Evaluation}

Move the virtual human to the best standing position, and quantitatively evaluate the accessibility of 8 screws according to the evaluation rules described in 3.4.2 through the developed accessibility evaluation tool, as shown in Fig. 9. In this process, there is no need to adjust the posture of the virtual human. 
10

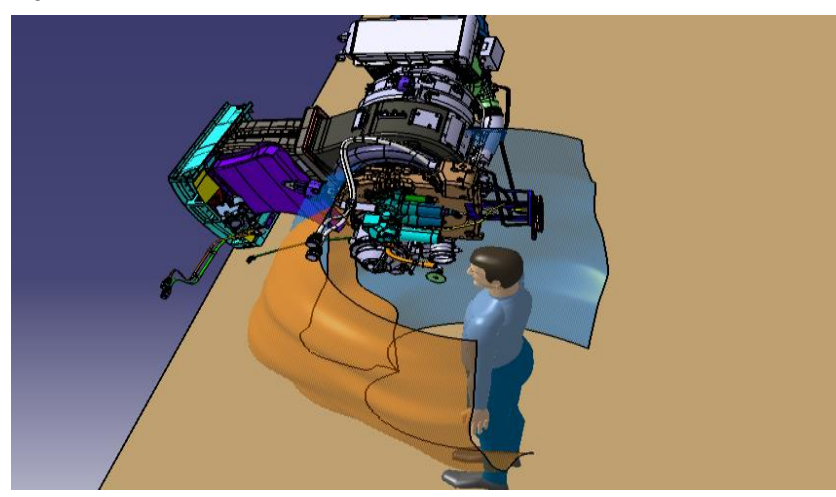

(a) Import the largest envelope

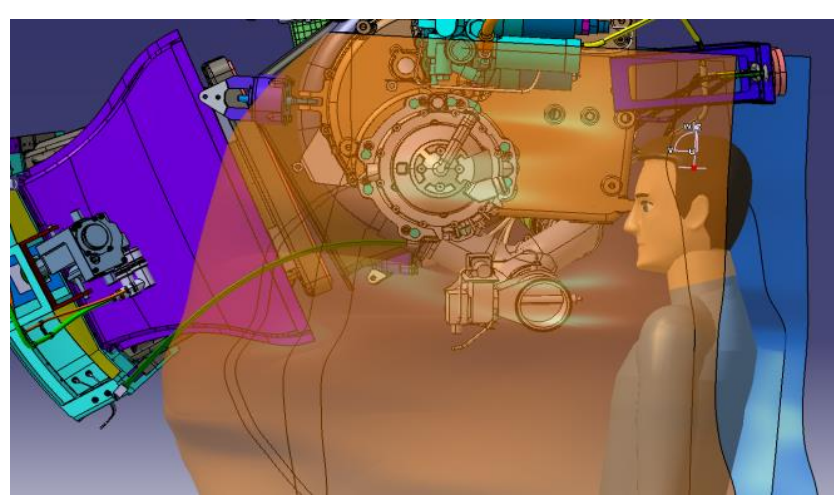

(b) Import the envelope surface from large to small and check the accessibility of bolts in turn (Fig (b) shows two bolts with accessibility score of 2)

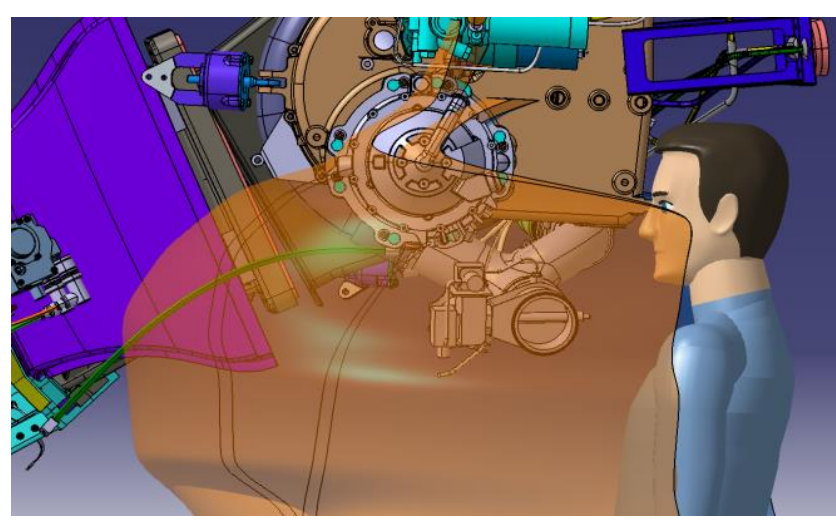

(c) Two bolts with accessibility score of 5

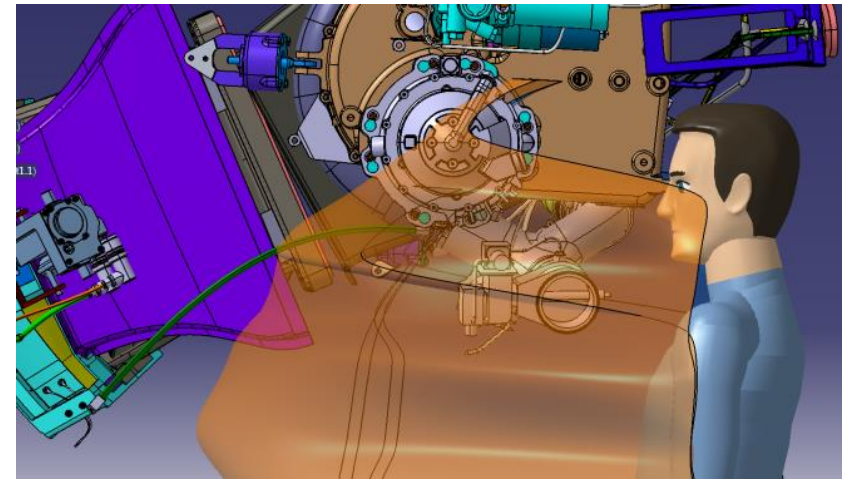

(d) Two bolts with accessibility score of 6

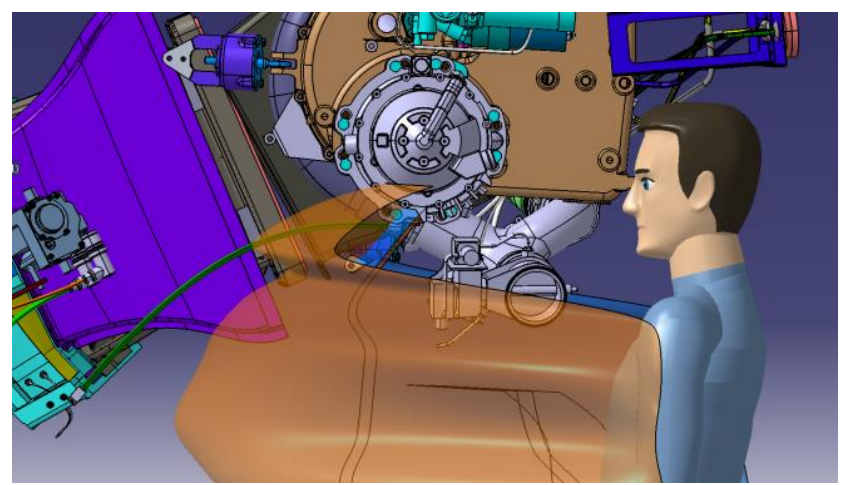

(e) Two bolts with accessibility score of 7

Fig. 9 The accessibility evaluation carried out through the developed accessibility evaluation tool

The accessibility scores of 8 bolts are shown in Table 5, of which two bolts score 2 points and two bolts score 5 points. Therefore, although some bolts are accessible, the posture of maintenance personnel is not comfortable, which is consistent with the results obtained by dynamic virtual maintenance simulation and physical prototype maintenance simulation.

Table 5 Accessibility score of 8 fastening bolts around starter

\begin{tabular}{|c|c|c|c|c|}
\hline Number & 1 & 2 & 3 & 4 \\
\hline Score & 7 & 7 & 6 & 6 \\
\hline Number & 5 & 6 & 7 & 8 \\
\hline Score & 5 & 5 & 2 & 2 \\
\hline
\end{tabular}

Open the reach envelope in DELMIA, as shown in Fig 10. The reach envelope shows the maximum reachable range of human arms. After verification, all 8 bolts are reachable. The result of accessibility evaluation of proposed method is consistent with the evaluation result of DELMIA reach envelope, but DELMIA reach envelope can only judge whether it is reachable or not. In this case, although the two most 
distal bolts can be reached, they are very uncomfortable during maintenance. Such a design will highly affect the efficiency and effect of maintenance, but the accessibility evaluation results of DELMIA can not reflect this maintainability defect.

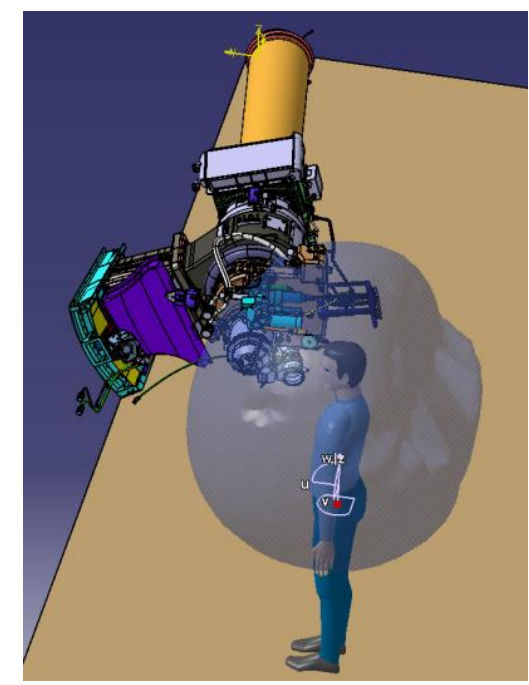

Fig. 10 The accessibility evaluation carried out through the reach envelope in DELMIA

\section{Conclusion}

In fact, the method of evaluating the accessibility in virtual maintenance is to establish a connection between comfort and accessibility, and quantify and refine the evaluation of accessibility through comfort. Specifically, a scientific and quantitative accessibility evaluation method is constructed by constructing the D-H parametric kinematics model of human body and dividing and scoring the joint angles through RULA, which solves the problems of high cost, strong subjectivity and low granularity in virtual maintenance accessibility evaluation. In order to improve the evaluation efficiency and facilitate the use of maintenance personnel, the corresponding reachable envelope surface model is built for each accessibility score, and an accessibility evaluation tool which can be integrated into DELMIA and CATIA is developed. At present, the tool has been used in research units such as AVIC First Aircraft Design and Research Institute and AECC Hunan Aviation Powerplant Research Institute, and the feedback is good. The future research direction of this method may consider the influence of obstacles on accessibility.

\section{Acknowledgement}

The authors would like to express our gratitude to all those who provided assistance during the writing of the paper. We are also pleased to acknowledge Mr. Chengzhang Chen for searching for a detailed database of parameters for human body. Finally, we are extremely grateful for Mr. Chao Dai for proofreading the article and offering a proposal regarding the outline of the article.

\section{Declarations}

Funding Not applicable.

Conflicts of interest The authors declare no conflicts of interest.

Data availability Not applicable.

Code availability Not applicable.

Ethics approval Not applicable.

Consent to participate Not applicable.

Consent for publication All the authors have reviewed and agreed to publish this research work.

Contributions Conceptualization: Dong Zhou and Hongduo $\mathrm{Wu}$; methodology and investigation: Hongduo $\mathrm{Wu}$, Ziyue Guo, Qidi Zhou; modelling and visualization: Hongduo $\mathrm{Wu}$, Yuning Liang; case verification: Dong Zhou, Hongduo $\mathrm{Wu}$; original draft preparation: Hongduo Wu, Ziyue Guo.

\section{References}

[1] Yingxiao Qin, Mingde Zhou, Yong Yan, et al. (2002). Introduction to reliability, maintainability and supportability. Beijing: National Defense Industry Press.

[2] Lu Chen, Dandong Jiang. (2003). Research on evaluation model and implementation algorithm of product maintainability. China Mechanical Engineering 14(021), 1857-1859.

[3] Guo, Z. , Zhou, D. , Zhou, Q. , X Zhang, \& Hao, A. . (2020). Applications of virtual reality in maintenance during the industrial product lifecycle: a systematic review. Journal of Manufacturing Systems, 56, 525-538.

[4] O. Battaia, A. Dolgui, S.S. Heragu, S.M. Meerkov, M.K. Tiwari (2018). Design for manufacturing and assembly/disassembly: joint design of products and production systems. International Journal of Production Research, 56(24), 7181-7189.

[5] Abshire, K. J. , Barron, M. K. (1998). Virtual maintenance real-world applications within virtual environments. IEEE. IEEE. 132-137

[6] Fern, T. , Marcelino, L. , Wimalaratne, P. . (2002). Constraint-based Immersive Virtual Environment for Supporting Assembly and Maintenance Tasks.

[7] Marcelino, L. , Murray, N. , Fernando, T. . (2003). A constraint manager to support virtual maintainability. Computers \& Graphics, 27(1), 19-26.

[8] Kallmann, M. , Thalmann, D. . (2002). Modeling behaviors of interactive objects for real-time virtual environments. Journal of Visual Languages \& Computing, 13(2), 177-195.

[9] Vujosevic, R. , Ianni, J. . (1997). A Taxonomy of Motion Models for Simulation and Analysis of Maintenance Tasks.

[10] Wampler, J. L. , Bruno, J. M. , Blue, R. R. , \& Hoebel, L. J. . (2003). Integrating maintainability and data development. Reliability \& Maintainability Symposium. IEEE.

[11] Wenju Wang, Guangyao Lee. (2011). Research and development of virtual maintenance simulation technology. Journal of system simulation (9), 1751-1757.

[12] Zhanhai Wang, Qinggang Zhai. (2009). Application of DELMIA ergonomics in virtual maintenance of aircraft. Electromechanical technology, 032, 36-44,53.

[13] Songshan Wang, Jianping Hao. (2004). Virtual maintenance prototype modeling based on interactive features. Computer simulation, 21(12).

[14] Xinxing Lee, Jianping Hao, Hui Liu. (2005). Design and implementation of maintenance motility in virtual 
[15] LV Chuan, Xu Hongqiang, Ma Lin, et al. (2005). Maintenance simulation driven by "hybrid control" virtual human. Journal of Beijing University of Aeronautics and Astronautics, 31(005), 544-547.

[16] Zhou Dong, LV Chuan. (2006). Modeling and control implementation of virtual maintenance simulation. Journal of Beijing University of Aeronautics and Astronautics, 32(007), 843-846.

[17] Guo, Z. , D Zhou, Zhou, Q. , Mei, S. , Zeng, S. , \& D Yu, et al. (2020). A hybrid method for evaluation of maintainability towards a design process using virtual reality. Computers \& Industrial Engineering, 140(Feb.), 106227.1-106227.14.

[18] Yang Yingyan, Jiang Keyi, Li Benwei, \& Wang Yonghua. (2009). Research progress of virtual maintenance simulation. Journal of Naval Institute of Aeronautical Engineering (04), 413-418.

[19] GJB / Z91, Maintainability design technical manual.

[20] Yongli Yu, Jianping Hao, Xiaodong Yang. (1998). Current situation and development trend of maintainability visual design and analysis technology. China Mechanical Engineering (12), 41-44.

[21] (2008). Literature: hardware/software. Rubber World.

[22] Yang Yuhang, Li Zhizhong, \& Zheng Li. (2005). Summary of virtual maintenance research. Journal of system simulation (09), 148-152 + 155 .

[23] Pan Yong (2004). Reliability maintainability supportability engineering software CARMES successfully applied to Shenzhou V manned spacecraft. Reliability and Environmental Test of Electronic Products (1), 19-19.

[24] Liu Kui. (2003). Take off! Start with numbers - AVIC first aircraft research institute has designed the first full-scale electronic prototype of aircraft. Chinese Computer Users (38), 33-33.

[25] Guo, Z. , Dong, Z. , Chen, J. , Jie, G. , \& Zeng, S. . (2018). Using virtual reality to support the product's maintainability design: immersive maintainability verification and evaluation system. Computers in Industry, 101, 41-50.

[26] W Karwowski, W S Marras. (2003). Occupational Ergonomics. CRC Press.

[27] Mcatamney, L. Nigel, C. E. . (1993). Rula: a survey method for the investigation of work-related upper limb disorders. Applied Ergonomics, 24(2), 91-99.

[28] Dohyung, Kee, and, Waldemar, \& Karwowski. (2001). Luba: an assessment technique for postural loading on the upper body based on joint motion discomfort and maximum holding time-sciencedirect. Applied Ergonomics, 32(4), 357-366.

[29] Qianxiang Zhou, Gui Cai, Hongwei Wang, Zhifeng Zhao. (2009). The latest development of ergonomic virtual human. Computer Simulation, 026(005), 6-9.

[30] Xiaotao Yang, Kejian Yang, Chunxiang Yan. (2008). Research on geometric modeling and motion control of virtual human. Computer and Digital Engineering, 36(008), 132-135.

[31] Yi Gan, Junlei Wang, Fujia Sun. (2014). D-H parameter optimization design of $6 \mathrm{R}$ robot based on given workspace. China Mechanical Engineering, 25(022), 30033007,3011 .

[32] GB 10000-88. S. Human dimensions of Chinese adults (State Bureau of Technical Supervision Press, Beijing 1988). 